\title{
Lithium-Doped 3D Covalent Organic Frameworks: High-Capacity Hydrogen Storage Materials**
}

\author{
Dapeng Cao, Jianhui Lan, Wenchuan Wang, * and Berend Smit*
}

It has become increasingly clear that hydrogen as a viable energy carrier may play an important role in future energy plans. However, development of reasonably safe and reversible hydrogen storage materials for transportation is still a great challenge. ${ }^{[1]}$ Using mesoporous carbon materials, it has to date been difficult to meet the storage capacity target of $6 \mathrm{wt} \%$ set by the US Department of Energy for hydrogen fuel-cell vehicles. A pressing task is to seek new materials for efficient hydrogen storage. An important step forward was the introduction of metal-organic frameworks (MOFs). ${ }^{[2]}$ Some MOFs, for example MOF-177 and MOF-5, have high BET specific surface areas (SSAs), and the strong London dispersion between linkers and connectors with hydrogen makes them attractive materials for hydrogen adsorption and storage. $^{[3,4]}$ However, MOFs still behave poorly at room temperature and cannot reach the target for practical use.

Schlapbach and Zuttel ${ }^{[1]}$ pointed out that high SSA is a key factor to store hydrogen efficiently. Recently, a family of three-dimensional (3D) covalent organic frameworks (COFs) was synthesized by Yaghi and co-workers. ${ }^{[5]}$ The crystalline 3D COFs were synthesized by self-condensation and cocondensation reactions of the rigid molecular building blocks tetrahedral tetra(4-dihydroxyborylphenyl)methane (TBPM) or its silane analogue (TBPS) and triangular hexahydroxy triphenylene (HHTP; Figure 1). The $\mathrm{B}_{3} \mathrm{O}_{3}$ and $\mathrm{C}_{2} \mathrm{O}_{2} \mathrm{~B}$ rings of these materials are covalently connected to form very stable nanoporous frameworks. These COFs possess not only extremely high surface areas but also extraordinarily low densities. For example, the BET SSAs of COF-102 and COF103 reach 3472 and $4210 \mathrm{~m}^{2} \mathrm{~g}^{-1}$, and the densities are 0.41 and $0.38 \mathrm{~g} \mathrm{~cm}^{-3}$, respectively. COF-105 and COF-108 have even lower densities of 0.18 and $0.17 \mathrm{~g} \mathrm{~cm}^{-3}$, respectively. Impor-

[*] Prof. Dr. D. Cao, J. Lan, Prof. W. Wang

Division of Molecular and Materials Simulation

Key Lab for Nanomaterials, Ministry of Education of China

Beijing University of Chemical Technology, Beijing 100029 (China)

Fax: (+86) 10-6442-7616

E-mail:wangwc@mail.buct.edu.cn

Homepage: http://www.ms.buct.edu.cn

Prof. Dr. B. Smit

Department of Chemical Engineering and Department of Chemistry University of California, Berkeley, CA 94720-1462 (USA)

E-mail: berend-smit@berkeley.edu

[***] This work is supported by NSF of China (20776005, 20736002), Beijing Novel Program (2006B17), National Basic Research Program of China (2007CB209706), NCET Program (NCET-06-0095) from the MOE, Chemical Grid Program from BUCT. We thank Prof. J. Long at UC Berkeley for his comments.

(9) Supporting information for this article is available on the WWWW under http://dx.doi.org/10.1002/anie.200900960.

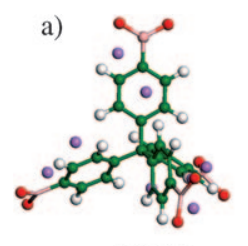

TBPM

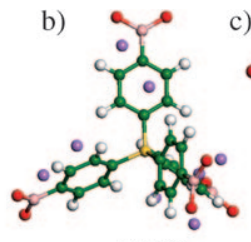

TBPS

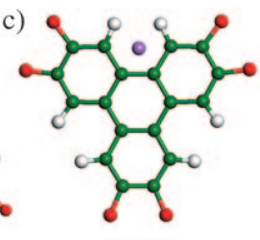

HHTP

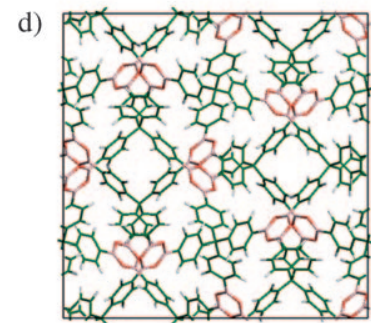

COF-102

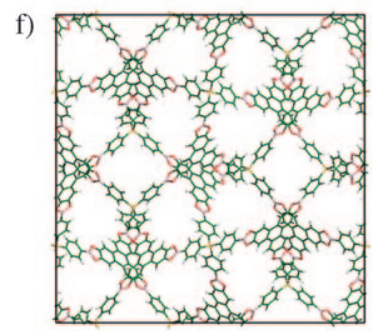

COF-105

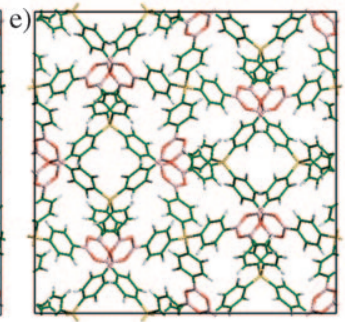

COF-103

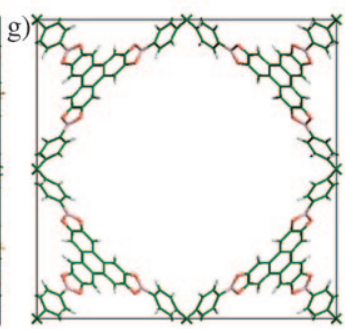

COF-108
Figure 1. a-c) Li-doped building blocks and $d-g$ ) unit cells of 3D covalent organic frameworks. $\mathrm{H}$ atoms linked to oxygen in $(\mathrm{a}-\mathrm{c})$ are omitted for clarity. The scheme of Li doping on 3D COFs is determined from calculations (see the Supporting Information for details). Li violet, $\mathrm{H}$ white, B pink, $\mathrm{C}$ green, $\mathrm{O}$ red, Si yellow.

tant is that the entire material is accessible and an adsorbed molecule "sees" all the atoms of the framework. These impressive numbers make these materials ideal candidates for hydrogen storage. Herein we address the question of whether these materials have excellent hydrogen storage capacity at both cryogenic and room temperatures, and we introduce a multiscale theoretical method, ${ }^{[6]}$ which combines first-principles calculations and grand canonical Monte Carlo (GCMC) simulation, to evaluate the hydrogen-adsorption capacities.

The first-principles calculations were performed at the second-order Møller-Plesset (MP2) level of theory using the cc-PVTZ basis set to investigate the interaction between $\mathrm{H}_{2}$ and COFs. Then, the calculated potential energies were fitted to the force fields (FF), yielding the FF parameters between $\mathrm{H}_{2}$ and COFs. Using the force fields, GCMC simulations were employed to predict the adsorption isotherm of $\mathrm{H}_{2}$ in COFs (see the Supporting Information). 
To ensure that our method is accurate enough to give correct predictions, the experimental isotherms of $\mathrm{Ar}$ in $\mathrm{COFs}^{[5]}$ were used as a benchmark to calibrate the accuracy of our method. Figure 2 shows that the calculated adsorption

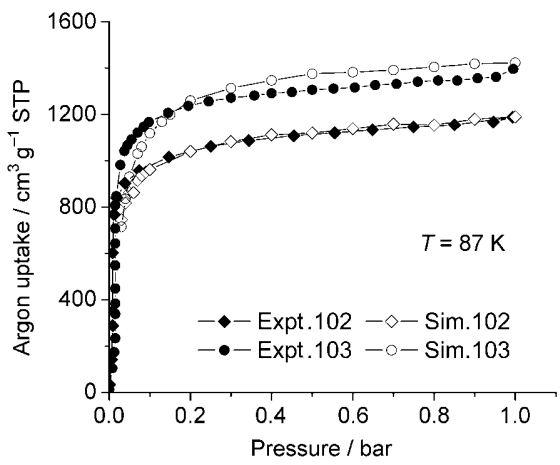

Figure 2. Comparison of the simulated and experimental adsorption isotherms of Ar on COF-102 and COF-103 at $T=87 \mathrm{~K}$.

isotherms of $\mathrm{Ar}$ in COF-102 and COF-103 at $T=87 \mathrm{~K}$ are in satisfactory agreement with the experimental data. This result is encouraging, in particular if we compare our approach with the results obtained by Garberoglio, ${ }^{[7]}$ who pointed out that the Dreiding and UFF force fields gave isotherms that deviate $25 \%$ from the experimental data. As we used the same multiscale procedure for $\mathrm{H}_{2}$ adsorption, the agreement of $\mathrm{Ar}$ isotherms with experiment gives us some confidence that our method is able to give reasonable predictions for $\mathrm{H}_{2}$.

Figure 3 shows the predicted $\mathrm{H}_{2}$ adsorption isotherms in COFs at $T=77 \mathrm{~K}$. Our results show that COF-105 and COF108 have the highest $\mathrm{H}_{2}$ gravimetric storage capacities, with uptakes of 18.05 and $17.80 \mathrm{wt} \%$, respectively, at $p=100 \mathrm{bar}$, owing to their high surface areas and free volumes. These values are very exciting compared to the highest reported hydrogen storage capacity of approximate $10 \mathrm{wt} \%$ for MOFs at similar conditions (MOF-177, ${ }^{[8]} 0.42 \mathrm{~g} \mathrm{~cm}^{-3} ; \mathrm{MOF}-5,{ }^{[9]}$ $\left.0.59 \mathrm{~g} \mathrm{~cm}^{-3}\right)$. The storage capacities of COF-102 and COF103 also reach 10.70 and $11.78 \mathrm{wt} \%$ at $p=100$ bar. Figure $3 \mathrm{~b}$ shows the excess gravimetric adsorption isotherms of $\mathrm{H}_{2}$ in COFs. We defined the excess amount as the amount of total adsorption minus the amount of hydrogen in the free volume in the bulk phase. The excess isotherms indicate that at $T=$ $77 \mathrm{~K}, \mathrm{COF}-105$ and COF-108 display optimal gravimetric adsorption capacities ( 10.31 and $10.26 \mathrm{wt} \%$ at $p=50 \mathrm{bar}$ ). On the basis of the above analysis, the COFs have exceeded MOFs, owing to their extraordinarily low densities, especially for COF-105 and COF-108. The low densities and the maximized fraction of surface sites ${ }^{[5]}$ make COFs the most promising candidates for hydrogen storage. Most recently, Han et al. ${ }^{[10]}$ and Klontzas et al. ${ }^{[11]}$ reported their predictions on the hydrogen storage capacity of COFs at $T=77 \mathrm{~K}$ by simulation. Their studies gave results very similar to ours, which shows that our quantum-mechanics-based multiscale method is valid in predicting the hydrogen storage capacity of COFs.

Owing to the poor hydrogen storage performance of traditional microporous materials at room temperature, we
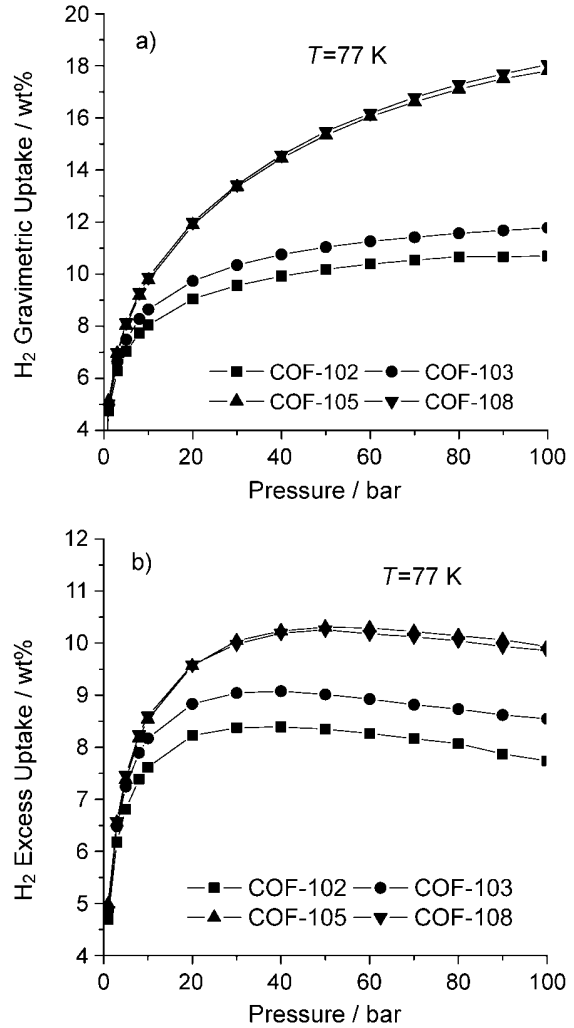

Figure 3. Computed $\mathrm{H}_{2}$ adsorption isotherms in COFs at $T=77 \mathrm{~K}$. a) Total gravimetric isotherms. b) Excess gravimetric isotherms.

also predicted the isotherms of $\mathrm{H}_{2}$ in COFs at $T=298 \mathrm{~K}$ (see the Supporting Information). Our results show that the $\mathrm{H}_{2}$ storage capacities of COF-105 and COF-108 reach 4.67 and $4.51 \mathrm{wt} \%$, respectively, at $p=100 \mathrm{bar}$, which is significantly higher than the reported capacities of MOFs. ${ }^{[12,13]}$

To improve storage capacity of hydrogen, Goddard and $\mathrm{Han}^{[14]}$ recommended that doping electropositive metals into MOFs might be a good strategy. Mulfort and Hupp ${ }^{[15]}$ used chemical reduction methods to obtain the Li-doped MOF, and they indeed found that the hydrogen adsorption capacity nearly doubled. Mavrandonakis et al. ${ }^{[16]}$ demonstrated that this enhanced adsorption is caused by the strong binding of $\mathrm{H}_{2}$ at the positively charged Li cations doped on MOFs. In light of these exciting reports, we further studied adsorption of hydrogen in Li-doped COFs at room temperature.

To gain information about the adsorption of $\mathrm{Li}$ in COFs, we first systematically performed first-principles calculations to study the doping details of single and multiple Li atoms in COFs. The widely used B3LYP/6-31g(d) method in the firstprinciples calculation was adopted for geometry optimizations, which is expected to give reliable adsorption sites. In our calculations, we placed eight Li atoms on the TBPM and TBPS building blocks, respectively, and the average binding energy per $\mathrm{Li}$ atom is approximately $-24.90 \mathrm{kcal} \mathrm{mol}^{-1}$. Moreover, we placed one $\mathrm{Li}$ atom on the HHTP building block, and the binding energy is approximately $-14.71 \mathrm{kcal}$ $\mathrm{mol}^{-1}$ (see Figure $1 \mathrm{a}$ to $\mathrm{c}$ ). In this scheme, all the Li atoms are positively charged with more than $0.3|\mathrm{e}|$ per Li atom. Our first-principles calculations indicated that this distribution 
scheme for $\mathrm{Li}$ doping in COFs is reasonable and acceptable, such that it should provide relatively reliable predictions of the hydrogen adsorption capacity of the Li-doped COFs. This scheme is determined based on the fact that only positively charged $\mathrm{Li}$ atoms contribute to the enhancement of hydrogen capacity, whereas the neutral $\mathrm{Li}$ atoms and anions do not. Furthermore, when the COFs are doped with multiple $\mathrm{Li}$ atoms, the adsorbed $\mathrm{Li}$ atoms may tend to form clusters, thus inhibiting the charge transfer from $\mathrm{Li}$ to $\mathrm{COFs}$ to some extent (see the Supporting Information for details).

It is essential to define the interactions between $\mathrm{Li}$ and $\mathrm{H}_{2}$ after the determination of the doping of $\mathrm{Li}$ atoms. The Mulliken and natural population analysis reveal that the quantity of charge transferred from $\mathrm{Li}$ atoms to COFs is mainly determined by two factors: the ratio and distribution of $\mathrm{Li}$ dopants. In the distribution scheme presented in Figure 1, the charges transferred from the doped $\mathrm{Li}$ atoms to the COFs are in the range from 0.3 to $0.5|\mathrm{e}|$ per $\mathrm{Li}$ atom. Previous studies $^{[16,17]}$ found that the strong affinity of the positively charged $\mathrm{Li}$ atom for $\mathrm{H}_{2}$ is due to the formation of a dative bond between the electrons of the $\mathrm{H}_{2}$ $\sigma$ bond and the empty $\mathrm{Li} 2 \mathrm{~s}$ orbital. When a $\mathrm{H}_{2}$ molecule is adsorbed near a Li atom doped on the HHTP building block as shown in Figure $1 \mathrm{c}$, the total charge transfer from $\mathrm{H}_{2}$ is about $0.06|\mathrm{e}|$. The interaction between $\mathrm{Li}$ and $\mathrm{H}_{2}$ was obtained from high-quality first-principles calculations (PW91/6-311g(d,p)). The FF parameters of the $\mathrm{Li}-\mathrm{H}_{2}$ interaction derived from our first-principles calculations are presented in the Supporting Information.

Figure 4 a presents the simulated adsorption isotherms of $\mathrm{H}_{2}$ in Li-doped COFs at $T=298 \mathrm{~K}$. We observed that the gravimetric storage capacities of $\mathrm{H}_{2}$ in Li-doped COF-105 and COF-108 are significantly higher than all the Li-doped MOFs. Among the four frameworks, COF-105 and COF-108 show more than double increase and reach 6.84 and $6.73 \mathrm{wt} \%$, respectively, at $T=298 \mathrm{~K}$ and $p=100$ bar. A comparison of the volumetric capacities of the doped and undoped COFs shown in Figure $4 \mathrm{~b}$ indicates that all four Li-doped COFs achieve an approximately doubled enhancement in volumetric adsorption capacity of $\mathrm{H}_{2}$ at $T=298 \mathrm{~K}$ and $p=100 \mathrm{bar}$ (25.98, 25.00, 13.75, and $13.57 \mathrm{~g} \mathrm{~L}^{-1}$ for Li-doped COFs 102 , 103,105 , and 108 , respectively) compared to corresponding undoped COFs. The excess isotherms of $\mathrm{H}_{2}$ in Li-doped COFs (Figure $4 \mathrm{c}$ and $\mathrm{d}$ ) indicate that Li-doped COF-102 and COF103 exceed COF-105 and COF-108 not only in the gravimetric but also in the volumetric adsorption capacities at room temperature. At $T=298 \mathrm{~K}$ the excess gravimetric and volumetric adsorption capacities of $\mathrm{H}_{2}$ in Li-doped COF-102 reach $4.25 \mathrm{wt} \%$ and $21.11 \mathrm{gL}^{-1}$, respectively. At present, this performance is excellent for a microporous material at room temperatures. This above observation is in agreement with the predictions from the first-principles calculations. ${ }^{[18]}$

The capacity for reversible storage of hydrogen is of practical importance. Although a small portion of the hydrogen cannot be released at the discharge pressure, the gravimetric delivery capacity of hydrogen in the Li-doped COF-108 still reaches $6.03 \mathrm{wt} \%$ at $T=298 \mathrm{~K}$ and $p=100 \mathrm{bar}$ (see the Supporting Information).

Our calculations show that COF materials are superior to MOF materials in hydrogen storage. To meet the requirements for practical use in hydrogen storage, we suggest doping these materials with $\mathrm{Li}$ atoms; the gravimetric adsorption capacities for hydrogen in Li-doped COF-105 and COF-108 reach 6.84 and $6.73 \mathrm{wt} \%$ at $T=298 \mathrm{~K}$ and $p=$ 100 bar. To our knowledge, these are the most promising candidates for hydrogen storage to date. Our results will need further experimental validation and will help guide the development of new porous materials for hydrogen storage.

Received: February 18, 2009

Revised: April 16, 2009

Published online: May 22, 2009 
Keywords: adsorption - computer chemistry . covalent organic frameworks $\cdot$ first principles $\cdot$ hydrogen

[1] L. Schlapbach, A. Zuttel, Nature 2001, 414, 353.

[2] M. Eddaoudi, J. Kim, N. L. Rosi, D. T. Vodak, J. Wachter, M. O'Keeffe, O. M. Yaghi, Science 2002, 295, 469.

[3] X. Zhao, B. Xiao, A. J. Fletcher, K. M. Thomas, D. Bradshaw, M. J. Roesseinsky, Science 2004, 306, 1012.

[4] A. Kuc, T. Heine, G. Seifert, H. A. Duarte, Chem. Eur. J. 2008, 14, 6597.

[5] H. M. El-Kaderi, J. R. Hunt, J. L. Mendoza-Cortes, A. P. Cote, R. E. Taylor, M. O'Keeffe, O. M. Yaghi, Science 2007, 316, 268.

[6] J. Lan, D. Cheng, D. Cao, W. Wang, J. Phys. Chem. C 2008, 112, 5598.

[7] G. Garberoglio, Langmuir 2007, 23, 12154.

[8] H. Furukawa, M. A. Miller, O. M. Yaghi, J. Mater. Chem. 2007, $17,3197$.
[9] S. S. Kaye, A. Dailly, O. M. Yaghi, J. R. Long, J. Am. Chem. Soc. 2007, 129, 14176.

[10] S. S. Han, H. Furukawa, O. M. Yaghi, W. A. Goddard III, J. Am. Chem. Soc. 2008, 130, 11580.

[11] E. Klontzas, E. Tylianakis, G. E. Froudakis, J. Phys. Chem. C 2008, 112, 9095.

[12] N. L. Rosi, J. Eckert, M. Eddaoudi, D. T. Vodak, J. Kim, M. O'Keeffe, O. M. Yaghi, Science 2003, 300, 1127.

[13] A. G. Wong-Foy, A. J. Matzger, O. M. Yaghi, J. Am. Chem. Soc. 2006, $128,3494$.

[14] S. S. Han, W. A. Goddard III, J. Am. Chem. Soc. 2007, 129, 8422.

[15] K. L. Mulfort, J. T. Hupp, J. Am. Chem. Soc. 2007, 129, 9604.

[16] A. Mavrandonakis, E. Tylianakis, A. K. Stubos, G. E. Froudakis, J. Phys. Chem. C 2008, 112, 7290.

[17] J. G. Vitillo, A. Damin, A. Zecchina, G. Ricchiardi, J. Chem. Phys. 2005, 122, 114311.

[18] Y. J. Choi, J. W. Lee, J. H. Choi, J. K. Kang, Appl. Phys. Lett. 2008, 92, 173102. 